\title{
Forecasting Stock Exchange Using Soft Computing Techniques
}

\author{
Zainab Rahamneh, Mohamed Reyalat, ${ }^{1}$ Alaa Sheta ${ }^{2}$ Sultan Aljahdali ${ }^{3}$
}

\begin{abstract}
The financial industry is becoming more and more dependent on advanced computer technologies in order to maintain competitiveness in a global economy. Fuzzy logic represents an exciting technology with a wide scope for potential applications. There is a growing interest both in the field of fuzzy logic computing and in the financial world in explaining the use of fuzzy logic to forecast the future changes in prices of stocks, exchange rates, commodities, and other financial time series. Fuzzy algorithms are intensively used for the identification of dynamic models, combining both numerical and heuristic knowledge. Fuzzy logic provides a remarkably simple way to draw definite conclusions from vague, ambiguous or imprecise information. In this paper, we are investigating the ability of Fuzzy logic (FL) to tackle the financial time series forecasting problems. Experimental results on set of applications indicated that fuzzy logic can effectively solve these types of problems. In order to examine the effectiveness of fuzzy logic applied to forecasting, the comparison with Artificial Neural Networks (ANNs) is performed.
\end{abstract}

\section{Introduction}

Forecasting is the process of producing a set of outputs by given a set of inputs. The variables are normally historical data. Basically, forecasting assumes that future occurrences are based, at least in part, on presently observable or past events. It assumes that some aspects of the past patterns will continue into the future. Past relationships can then be discovered through study and observation. The basic idea of forecasting is to find an approximation of mapping between the input and output data in order to discover the implicit rules governing the observed movements. The need for forecasting is increasing as management attempts to decrease its dependence on chance and become more scientific in dealing with its environment. Forecasting practice has improved over time. For example, error in weather forecasting has decreased; by 1997 forecasters had correctly predicted $59 \%$ of tornados before they touched the ground [6].

1 M. Reyalat and A. Rahamneh are Teaching Assistants with the Information Technology Department, Prince Abdullah Bin Ghazi Faculty of Science and Information Technology, AlBalqa Applied University, Salt, Jordan. (zainab@yahoo.com, reyalat_bau@yahoo.com)

2 A. F. Sheta is a Professor of Computer Science and Engineering, College of Computers and Information Systems, Taif University, Taif, Saudi Arabia (asheta@tu.edu.sa)

$3 \mathrm{~S}$. H. Aljahdali is the Dean of the College of Computers and Information Systems, Taif University, Taif, Saudi Arabia (aljahdali@tu.edu.sa)
Financial time series forecasting is one of the most challenging applications of modern time series forecasting. Because financial time series are inherently noisy, nonstationary, and deterministically chaotic, there is no complete information that could be obtained from the past behavior of financial markets to fully capture the dependency between the future price and the past records [4]. Therefore, the financial industry is becoming more and more dependent on advanced computer technologies in order to maintain competitiveness in a global economy.

In this paper, our objective is to automate the process of developing forecasting model. The basic objective of the forecasting model is the construction of models from data that successfully mimic the relationships among data. This can be formulated as a supervised learning problem, where the goal is to model the relation between a set of input variables, and one or more output variables, which are considered dependent on the inputs. Once built, a model can take new inputs and produce a forecasting of the corresponding outputs. For financial time series purposes our interest lies in the application of these methods to system identification and forecasting systems.

\section{Fuzzy Logic (FL)}

In 1965, Lotfi A. Zadeh of the University of California at Berkeley invented the Fuzzy Sets, $[24,25]$ which laid out the mathematics of fuzzy set theory and, by extension, fuzzy logic. Since the last decade, the research on fuzzy sets and systems has drawn more and more attention. In fact, fuzzy modeling $[24,14]$ is now one of the most famous ways in dealing with nonlinear, uncertain and complex systems such as signal processing and mechanical control [17, 21, 11]. It has two important advantages: firstly, it imitates the human reasoning process using linguistic terms, which enables its comprehensibility and transparency; and secondly it is a universal modeling technique $[20,3,5,26]$ that can approximate any nonlinear complex system with specified arbitrary accuracy.

Application of fuzzy logic as an advance trading technology exploiting machine intelligence for financial time series market forecasting is an interesting area attracting much research effort worldwide. Application of fuzzy logic to stock market efficiency testing in the thesis context is an especially interesting topic considering the fact that it is an emerging market and there is little research in this area. In decisionmaking, fuzzy systems include fuzzy logic and trading rules provided by traders. A simple model of a fuzzy financial sys- 
tem may consist of one or more inputs (e.g. trend, stock price and volatility), one output (e.g., desired financial pattern), and a few fuzzy rules expressing the relationships between them. A fuzzy system fuzzifies inputs, creates membership functions, defines associations between the input and output variables in a fuzzy rule base, and then translates fuzzy outputs into financial recommendations. The process and theory underlying application of fuzzy systems was studied by $[18,8]$. Practical applications of fuzzy systems include systems for stock selection [22], foreign exchange trading [23], etc. fuzzy logic is also used to improve the effectiveness of neural networks by incorporating structured knowledge about financial markets, including rules provided by traders, and explaining how the output or trading recommendations were derived[7].

\section{Artificial Neural Network (ANN)}

Neural networks were developed as an attempt to realize simplified mathematical models of brain-like systems. The key advantage is their ability to learn from examples instead of requiring an algorithmic development from the designer. Due to the ability of neural networks to forecast future values, they are used to financially asses outcomes such as stock prices based on the past history of financial data including stock prices, financial indicators, and financial statistics. The networks are used in the predication and trading of stocks, indices, futures, options, and other tradable securities. They are also helpful in forecasting interest values or other economics measures $[10,13,16]$, forecast stock price indices and derivative securities [12], and predict exchange rates [15]. In [19] author show a novel neural-network-based method of time series forecasting to forecast stock price indices. Neural networks can be implemented as neuro-fuzzy networks which combine the advantages of both fuzzy reasoning and neural networks.

\section{Evaluation Criteria}

We considered the Variance Accounted- For (VAF) as a measure of performance for the proposed FL and ANNs models. The VAF (Variance Accounted For) used to assess the quality of a model, by comparing the true output with the output of the proposed models.

The VAF is computed as follows:

$$
V A F=\left[1-\frac{\operatorname{var}\left(y_{1}-y_{2}\right)}{\operatorname{var}\left(y_{1}\right)}\right] \times 100 \%
$$

The VAF of two equal signals is $100 \%$. If the signals differ, $\mathrm{VAF}$ is lower.

\section{Amman Stock Exchange (ASE)}

The ASE was established in March 1999 as a non-profit, private institution with administrative and financial autonomy. It is authorized to function as an exchange for the trading of securities. The exchange is governed by a seven-member board of directors. A chief executive officer oversees day-to-day responsibilities and reports to the board. The ASE membership is comprised of Jordan's 52 brokerage firms [9].

In this problem, we explored the problem of predicting the closing Price of each company based on the previous history of that company over a period of time. We used two approaches for this which they are: 1) Fuzzy Logic Model for ASE 2) Neural Networks Model for ASE. The data series was used to develop two time series model based FL and NNs. The models takes the inputs $x_{1}, x_{2}, x_{3}$ to determine the output predicted closing prices $y$.

- $x_{1}$ : Closing price (price of the last fulfilled trade during the day).

- $x_{2}$ : Highest price paid during the day.

- $x_{3}$ : Lowest price paid during the day.

- $y$ : Predicted closing price.

\subsection{Proposed Fuzzy Logic Model for ASE}

We solved this problem using FMID toolbox that run under MATLAB [1, 2]. The setting parameter for this toolbox is shown in Table 1. The results that provided by using Fuzzy Logic, are shown in Figure 3 and Figure 2 which represent the training and testing outputs respectively. The ASE closing price forecast error is shown in Figure 3. The variance between actual values and estimated values are: 99.9892. This model

Table 1. Parameter Setting for Fuzzy Logic Model.

\begin{tabular}{|l|l|}
\hline Parameter & Value \\
\hline \hline Number of clusters & 3 \\
\hline Fuzziness parameter & 2 \\
\hline Termination criterion & 0.01 \\
\hline Type of antecedent & 1 \\
\hline Type of consequent & 1 \\
\hline
\end{tabular}

was generated from 200 data samples. It has 3 inputs and 1 output. The sampling period is $1 \mathrm{~s}$. The termination tolerance of the clustering algorithm was 0.01 , and the random initial partition was generated with seed equal to 210408 . The set of rules which decsibe the system is given as:

1. If $x_{1}$ is $A_{11}$ and $x_{2}$ is $A_{12}$ and $x_{3}$ is $A_{13}$ then $y(k)=0.15 x_{1}+1.64 x_{2}-0.818 x_{3}-0.00160$

2. If $x_{1}$ is $A_{21}$ and $x_{2}$ is $A_{22}$ and $x_{3}$ is $A_{23}$ then $y(k)=-0.687 x_{1}+0.196 x_{2}+1.50 x_{3}+0.00958$

3. If $x_{1}$ is $A_{31}$ and $x_{2}$ is $A_{32}$ and $x_{3}$ is $A_{33}$ then $y(k)=1.99 x_{1}-1.32 x_{2}+0.334 x_{3}+0.991$

Table 3. Cluster centers for ASE closing price.

\begin{tabular}{|c|rrr|}
\hline rule & $x_{1}$ & $x_{2}$ & $x_{3}$ \\
\hline 1 & $4.21 \cdot 10^{0}$ & $4.31 \cdot 10^{0}$ & $4.17 \cdot 10^{0}$ \\
2 & $5.37 \cdot 10^{0}$ & $5.41 \cdot 10^{0}$ & $5.28 \cdot 10^{0}$ \\
3 & $4.25 \cdot 10^{1}$ & $4.28 \cdot 10^{1}$ & $4.15 \cdot 10^{1}$ \\
\hline
\end{tabular}

\section{Proposed Neural Networks Model for ASE}

We solved this ASE prediction problem using Neural Networks toolbox under MATLAB. The setting parameter for 
Table 2. Consequent parameters for ASE closing price.

\begin{tabular}{|c|rrrr|}
\hline rule & $x_{1}$ & $x_{2}$ & $x_{3}$ & offset \\
\hline 1 & $1.50 \cdot 10^{-1}$ & $1.64 \cdot 10^{0}$ & $-8.18 \cdot 10^{-1}$ & $-1.60 \cdot 10^{-3}$ \\
2 & $-6.87 \cdot 10^{-1}$ & $1.96 \cdot 10^{-1}$ & $1.50 \cdot 10^{0}$ & $9.58 \cdot 10^{-3}$ \\
3 & $1.99 \cdot 10^{0}$ & $-1.32 \cdot 10^{0}$ & $3.34 \cdot 10^{-1}$ & $9.91 \cdot 10^{-1}$ \\
\hline
\end{tabular}

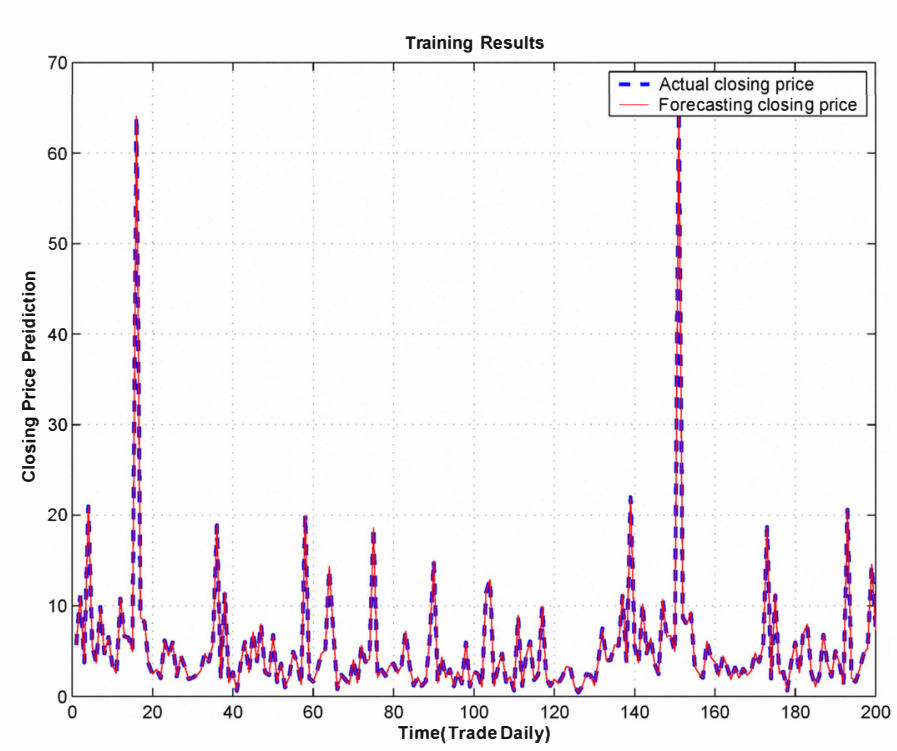

Figure 1. Forecasting of ASE Closing Price based on Fuzzy Logic Model- Training case.

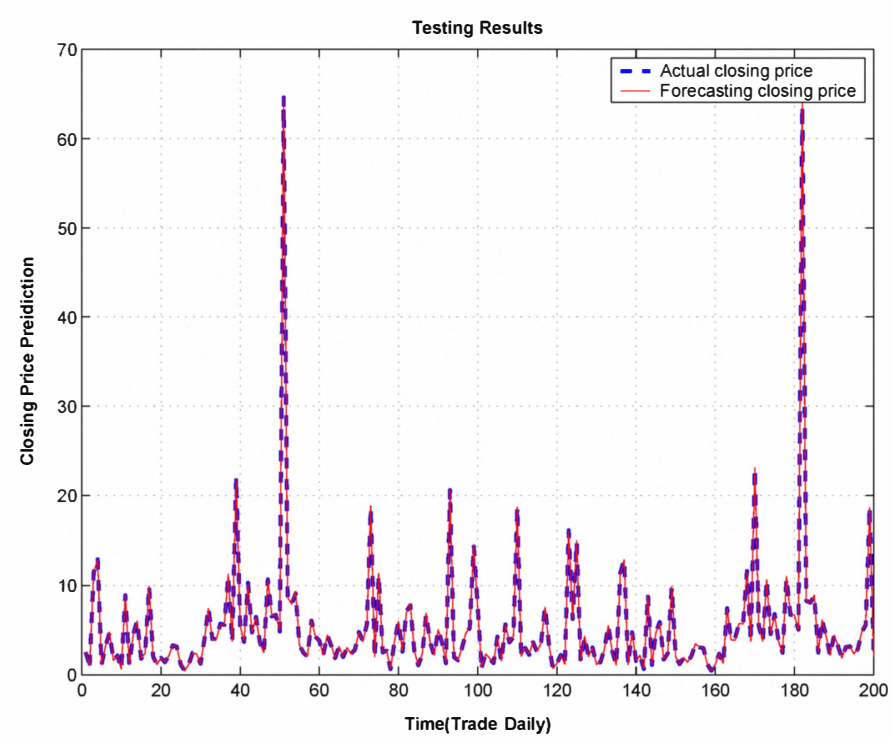

Figure 2. Forecasting of ASE Closing Price based on Fuzzy Logic Model- Testing case.

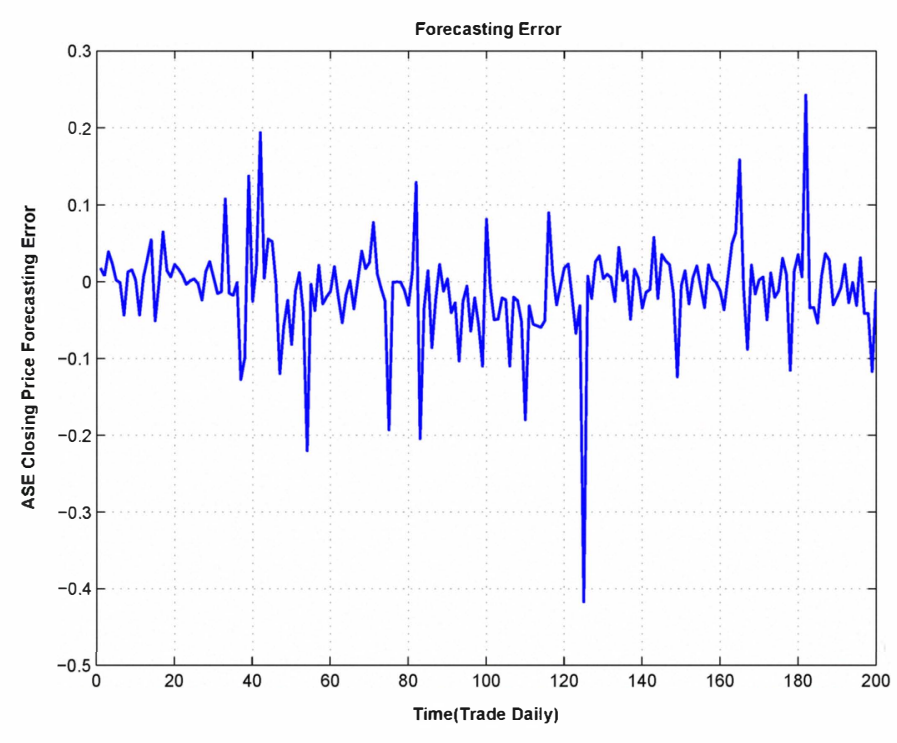

Figure 3. Forecasting error of ASE Closing Price based on Fuzzy Logic Model.

this toolbox is shown in Table 4. An artificial neuron has many inputs and only one output. Back propagation Neural Networks consist of many units-artificial neurons (processing elements) that are grouped in layers (see Figure 4). The Input layer gets the initial data (for example, historical prices of a stock), the Hidden layer calculates several interim values which are used to calculate output values(for example, predicated future values of the stock) in the Output layer. The results that provided by using Back Propagation Neu-

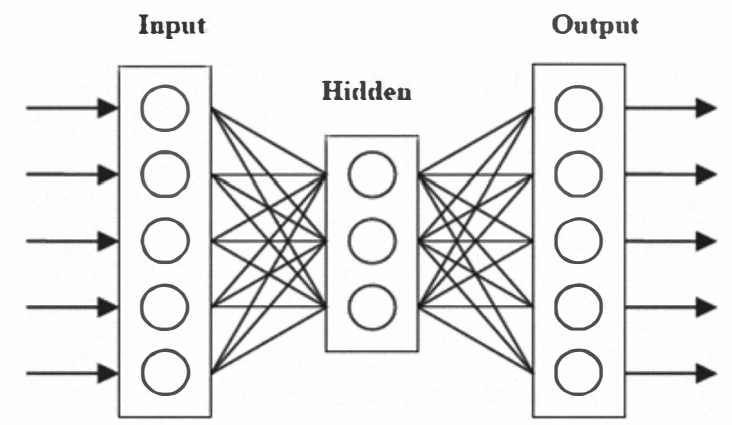

Figure 4. Fully Interconnected Network with One Hidden Layer ral Network are shown in Figures 5, 6 and 7 which represent 
the error convergence of the learning algorithms the output in both training and testing cases, respectively. The variance between actual values and estimated values are: 99.9775. The

Table 4. Neural Network Setting

\begin{tabular}{|l|l|}
\hline Parameter & Value \\
\hline \hline Number of Neuron in Input Layer & 30 \\
\hline Number of Neuron in Hidden Layer & 10 \\
\hline Number of Neuron in output Layer & 1 \\
\hline
\end{tabular}

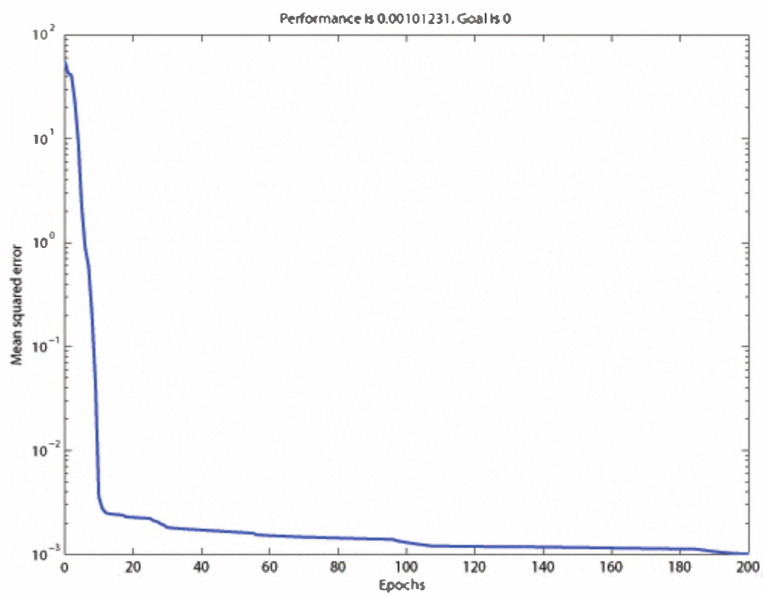

Figure 5. Convergence of the Neural Networks Model

computed Mean Squared Error (MSE) and VAF for various number of hidden layer units is shown in Table 5 . The results show that the best number of neurons in the hidden layer is ten (10).

Table 5. MSE and VAF for the ASE closing price forecasting using various number of neurons in the hidden layer

\begin{tabular}{|c|l|l|}
\hline $\begin{array}{c}\text { Number of neurons in } \\
\text { the Hidden layer }\end{array}$ & (MSE) & (VAF) \\
\hline \hline 5 & 0.00119305 & 99.9623 \\
\hline 7 & 0.00139321 & 99.9724 \\
\hline 10 & 0.00101231 & $\mathbf{9 9 . 9 7 7 5}$ \\
\hline 15 & 0.00128241 & 99.8765 \\
\hline
\end{tabular}

As shown in Table 6, Fuzzy Logic provides the suitable model compared to the Neural Network model, although the performance of both models looks quite similar.

\section{Conclusion and Future Work}

Forecasting modeling is the process of identifying a model of an unknown or complex process from numerical and historical data. Due to the inherent complexity of many real processes, conventional modeling techniques have proved to be too restrictive. In these instances more sophisticated modeling techniques are currently studied. In this paper, we explored the

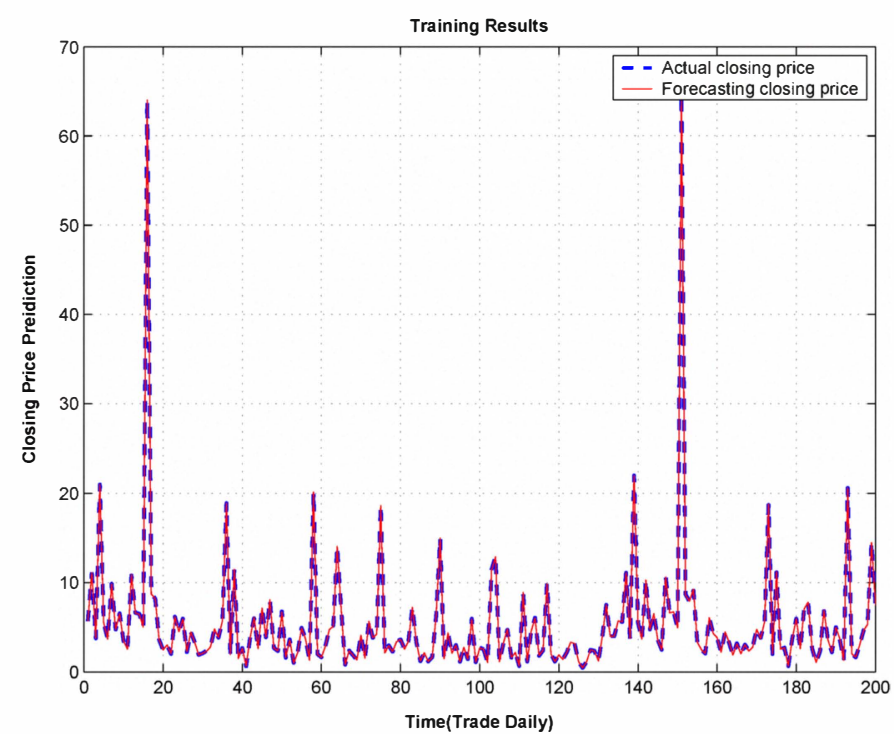

Figure 6. Forecasting of ASE Closing Price based on Neural Networks Model- Training case.

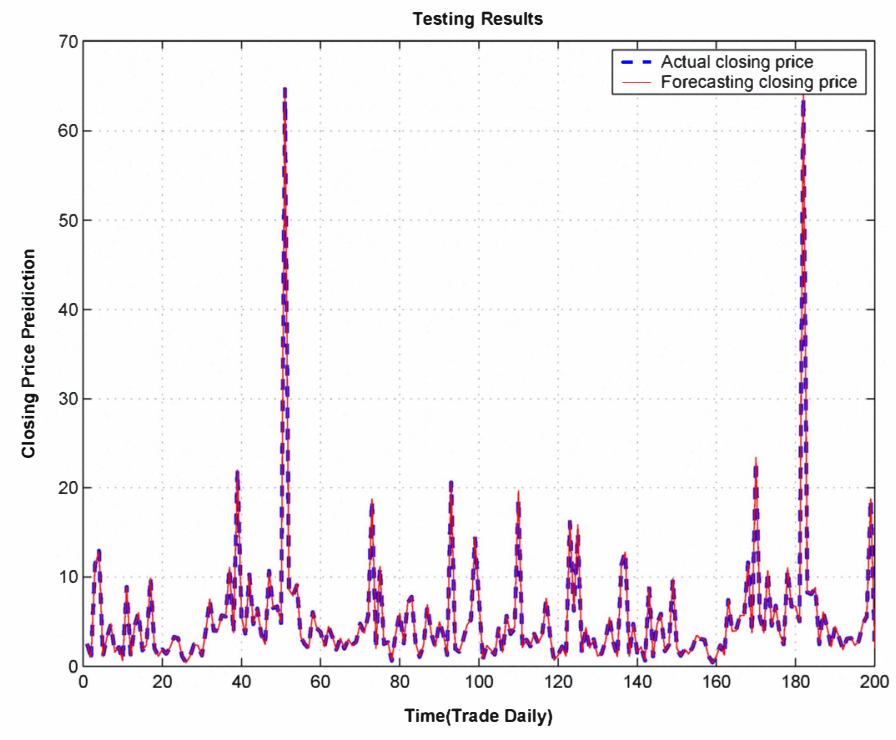

Figure 7. Forecasting of ASE Closing Price based on Neural Networks Model- Testing case.

Table 6. VAF for the three proposed models for ASE

\begin{tabular}{|l|l|}
\hline Model & Value of (VAF) \\
\hline \hline Fuzzy Logic model & 99.9892 \\
\hline Neural Networks model & 99.9775 \\
\hline
\end{tabular}


use of fuzzy logic modeling to solve financial time series forecasting problems. Fuzzy logic can overcome many problems encountered by traditional techniques. The generation of a fuzzy forecast model can be based both on expert knowledge and historical data. Fuzzy logic modeling systems offer the potential for a more flexible, less assumption approach to financial time series, and they have already been demonstrated as successfully substitutes for both the companies stocks and general index, and also as tools for the real time updating of financial time series forecasting models and especially for the multi-model approach.

\section{REFERENCES}

[1] R. Babus̃ka, Fuzzy Modeling and Identification Toolbox, Delft University of Technology, The Netherland, http://lcewww.et.tudelft.nl/bubuska, 1998.

[2] R. Babuska, J. A. Roubos, and H. B. Verbruggen, 'Identification of MIMO systems by input-output TS fuzzy models', in Proceedings of Fuzzy-IEEE'98, Anchorage, Alaska, (1998).

[3] Buckley93. J. Buckley, 'Sugeno type controllers are universal controllers', Fuzzy Sets and Systems, 3(5), 299-304, (1992).

[4] L.J Cao and E.H Tay Francis, 'Financial forecasting using support vector machines', in Neural Comput Appl, volume 10, pp. 184-192, (2001).

[5] J. L. Castro, 'Fuzzy logic controllers are universal approximators', IEEE Transactions on Systems, Man and Cybernetics, 25(4), (1995).

[6] Craft, 'The value of weather information services for nineteenth century great lakes shipping', American Economic Review, 88, 1059-1076, (1998).

[7] G.J Deboeck, Trading on the edge. Neural, Genetic, and Fuzzy Systems for chaotic financial markets, John Wiley and Sons, 1994.

[8] G.J. Deboeck, H. Green, M. Yoda, and G. S Jang, 'Adesign principles for neural and fuzzy trading systems'. International Joint Conference on Neural Networks, Beijing, (1992).

[9] Amman Stock Exchange, http://www.ammanstockex.com. Last Accessed April,2007, 2007.

[10] J. M. Hutchinson, T. Poggio, and A. W lo, A Nonparametric Approach to Pricing and Hedging Derivative Securities Via Learning Networks, 1994.

[11] R. Jensen, Combining rough and fuzzy sets for feature selection, Ph.D. dissertation, 2004.

[12] C.M. Kuan and T. Liu, 'Forecasting exchange rate using feedforward and recurrent neural networks', Journal of Applied Econometrics, 10(4), 347-364, (1995).

[13] E. Y. Li, 'Artificial neural networks and their business applications', Information and Managements, 27, 303-313, (1994).

[14] J. M. Mendel, 'Fuzzy logic systems for engineering: a tutorial', pp. 345-377. IEEE, (1995).

[15] M. Salah and F. Abid, 'Estimating term structure of interest rates: Neural network vs one factor parametric models', (2002).

[16] Y. Shachmurove and D. Witkowska, 'Dynamic interrelation among major world stock market: A neural network analysis', International Journal of Business, 6(1), 1-22, (2001).

[17] D Simon, 'Design and rule base reduction of a fuzzy filter for the estimation of motor currents.', IInternational Journal of Approximate Reasoning, 25(2), 145-167, (2000).

[18] M. Sugeno, Fuzzy Systems Theory and Its Applications, Tokyo Institute of Technology, 1992.

[19] Y. F. Sun, Y. C. Liang, W. L. Zhang, H. P. Lee W. Z. Lin, and L. J. Cao, 'Optimal partition algorithm of the rbf neural network and its application to financial time series forecasting', volume 14 , p. 3644, (2005).

[20] Wang.L.X and J. M.Mendel, 'Mendel. fuzzy basis functions, universal approximation, and orthogonal least-squares learning', IEEE Transactions on Neural Networks, 3(5), 807814, (1992).

[21] D. Willaeys and N. Malvache, 'Use of fuzzy model for process control', IEEE Proc. Int. Conf. Cybern. Soc, 942 946, (1979).
[22] F.S. Wong, P.Z. Wang, T.H. Goh, and B.K Quek, 'Fuzzy neural systems for stock selection', Financial Analyst Journal, (1992).

[23] H Yuize, 'Decision support system for foreign exchange trading'. First International Fuzzy Engineering Symposium, Yokohama., (1991).

[24] L. A. Zadeh, 'Fuzzy set', Information and Control, 8, 338353, (1965).

[25] L. A. Zadeh, 'Outline of a new approach to the analysis of complex systems and decision processes', IEEE Transaction on System, Man and Cybernetics, 3, 28-44, (1973).

[26] GX. J. Zeng and J. A. Keane, 'Approximation capability analysis of hierarchical takagi-sugeno fuzzy systems', pp. 1227-1232. IEEE International Conference on Fuzzy Systems, (2004). 